\title{
Light sedation or no-sedation in ICU: patient experience matters
}

\author{
Saurabh Mittal, Karan Madan, Anant Mohan, Vijay Hadda \\ Department of Pulmonary, Critical Care and Sleep Medicine, All India Institute of Medical Sciences, New Delhi, India
}

Dear Editor,

We have been using sedation for a long time in intensive care units for patients on mechanical ventilation. Sedation aims at facilitating the patient's ability to remain comfortably connected to the ventilator and minimize oxygen consumption. The strategies for sedation vary from deep sedation and light sedation to nosedation. For the last two decades, light sedation with daily interruption is being practiced by most ICUs.

A recently published article in the New England Journal of Medicine by Olsen et al. compared the strategies of no-sedation and light sedation in a randomized manner to assess its impact on mortality and various secondary outcomes [1]. The study reported no difference in mortality at 90 days, although an increase in thrombotic complications was reported in the light sedation group. Authors have explored multiple secondary outcomes, up to 90 days, including days free from coma/delirium and incidence of acute kidney injury. One crucial outcome, which needs to be studied in any study regarding sedation practices in the intensive care unit is the occurrence of post-traumatic stress disorder (PTSD) [2]. As clinicians, we tend to focus on survival benefits as primary out-

Correspondence: Saurabh Mittal Department of Pulmonary, Critical Care and Sleep Medicine, Ansari Nagar, New Delhi 110029, India. Tel. $+91.11 .26593488,+91.9968859414$.

E-mail: Saurabh_kgmu@yahoo.co.in

Key words: Sedation; ICU; PTSD.

Funding: None.

Conflict of interest: The authors declare no conflict of interest.

Contributions: All the authors made a substantive intellectual contribution, performed part of the experiments. All the authors have read and approved the final version of the manuscript and agreed to be accountable for all aspects of the work.

Ethics approval and consent to participate: Not applicable.

Received for publication: 23 May 2020.

Accepted for publication: 15 June 2020.

${ }^{\circ}$ Copyright: the Author(s), 2020

Licensee PAGEPress, Italy

Monaldi Archives for Chest Disease 2020; 90:1401

doi: 10.4081/monaldi.2020.1401

This article is distributed under the terms of the Creative Commons Attribution Noncommercial License (by-nc 4.0) which permits any noncommercial use, distribution, and reproduction in any medium, provided the original author(s) and source are credited. come while missing the issue of the patient experience. It is not known if a no-sedation strategy is associated with any change in the incidence of PTSD. ICU stay is a traumatic event in a patient's life, and a significant proportion of individuals fulfil criteria for PTSD after ICU discharge. Sedation plays a vital role in patients alertness and assessment of surroundings. Patients having wide variability in the consciousness level are more likely to be disoriented and horrified as compared to ones who are kept more awake. Secondly, during ICU stay the patient experiences different things such as invasive procedures on self as well as on other patients and sometimes has a near-death experience. The impact of these experiences is also likely to vary depending upon sedation level. Since the current trial has demonstrated no survival benefit of a no-sedation strategy, it becomes more critical to assess its effect on patient experience based outcomes such as the occurrence of depression and PTSD.

\section{References}

1. Olsen HT, Nedergaard HK, Strøm T, et al. Nonsedation or Light Sedation in Critically Ill, Mechanically Ventilated Patients. N Engl J Med 2020 19;382:1103-11.

2. Burki TK. Post-traumatic stress in the intensive care unit. Lancet Respir Med 2019 Oct 1;7:843-4. 